\title{
A Case Report of Primary Sjögren's Syndrome Presenting Oral Mucosal Pain
}

\author{
Ji-Young Lee, Jin-Seok Byun, Jae-Kwang Jung, Jae-Kap Choi \\ Department of Oral Medicine, School of Dentistry, Kyungpook National University, Daegu, Korea
}

Received May 11, 2021

Revised June 4, 2021

Accepted June 6, 2021
Correspondence to:

Jae-Kap Choi

Department of Oral Medicine, School of Dentistry, Kyungpook National University,

2175 Dalgubeol-daero, Jung-gu, Daegu

41940, Korea

Tel: $+82-53-600-7321$

Fax: +82-53-426-2195

E-mail: jhchoi@knu.ac.kr

https://orcid.org/0000-0001-6773-7507
Sjögren's syndrome (SS) is an autoimmune rheumatic disease characterized by the presence of lymphocytic infiltration of exocrine glands and other organs. Due to the rarity of SS, it is frequently overlooked by dentists as a cause of xerostomia. Clinicians should be aware that patients may have oral complications from SS including oral mucosal pain caused by frictional damage. Use of the latest diagnostic criteria approved by the American College of Rheumatology/European League against Rheumatism in 2016 for SS diagnosis provides the advantage of early identification of patients who have not yet developed dry mouth symptoms. A case of primary SS with the chief complaint of oral mucosal pain caused by mucosal damage associated with dry mouth is presented here. This case represents the importance of recognizing oral complications of xerostomia caused by SS, and illustrates use of the latest diagnostic criteria to diagnose the suspected symptoms by dental clinicians, who typically might be the first medical professional to encounter these symptoms.

Key Words: Diagnostic criteria; Oral complications; Sjögren's syndrome

\section{INTRODUCTION}

Sjögren's syndrome (SS) is an autoimmune rheumatoid disease characterized by lymphocyte infiltration into the exocrine glands and extra-glandular tissues. Oral and ocular dryness are its primary symptoms. The prevalence of primary SS varies based on estimates from literature; it is believed to be about $0.02 \%-0.10 \%$ in the United States [1]. It is found to be more than eight times prevalent in women than in men, and predominantly affects peri-menopausal individuals [2].

Dentists are often responsible for early differential diagnosis of SS since they frequently are the first medical personnel to encounter it due to the common development of oral symptoms [3]. However, since xerostomia is quite often induced by other causes like medication, radiation therapy, and aging it is not always easy for dentists to identify SS as a cause of xerostomia at the time of the first assessment. To avoid mistakes, patients with xerostomia need a thorough evaluation to determine the exact cause of xerostomia. Patients frequently present with oral mucosal pain as the chief complaint other than xerostomia, since hyposalivation makes them increasingly susceptible to oral ulcerative lesions or candidiasis. To make an accurate diagnosis, it is necessary not only to recognize sicca symptoms but also to notice the oral complications of SS.

Early and accurate diagnosis of SS is difficult since no diagnostic tests to confirm the disease are available yet. Proper application of reliable diagnostic schemes are essential for early diagnosis and prompt treatment. This would help improve the patient's quality of life through prevention of complications. Over the past 55 years, several diagnostic criteria have been proposed and further improved by experts to examine and evaluate signs and symptoms 
of primary SS. Certain diagnostic items with low sensitivity and/or specificity have been ruled out of the diagnostic criteria based on the outcome of further assessment. The latest diagnostic criteria, co-approved by the American College of Rheumatology/European League against Rheumatism (ACR/ EULAR) in 2016, introduced the weighted scoring system based on the diagnostic accuracy of previously used criteria. This includes the required presence of anti-SSA (Ro) antibodies or a labial salivary gland biopsy (LSGB) positive for glandular tissue inflammation. The additional strength of the current criteria is an easy application of the diagnostic criteria in clinical practice for early identification of the disease in SS patients prior to onset of systemic or sicca symptoms [4]. Disease diagnosis at an early age will enable patients to benefit from early treatment options.

In this article, we report a case of primary SS with chief complaint of oral mucosal pain caused by mucosal damage associated with dry mouth. This case was diagnosed following the 2016 ACR/EULAR diagnostic criteria and the characteristics of diagnostic criteria of primary SS are discussed in a chronological order.

\section{CASE REPORT}

A 69-year-old female patient visited the Department of Oral Medicine at Kyungpook National University Dental Hospital with chief complaint of oral pain in the bilateral buccal mucosa that began 5 months ago. During intraoral examination, bilateral ulcers surrounded by erythematous margins were found in her buccal mucosa (Fig. 1). She also reported onset of dryness of eyes and mouth that occurred post-menopause, 15 years back. Unstimulated whole saliva secretion test revealed a secretion rate of $0 \mathrm{~mL} / 10 \mathrm{~min}$.

From the patient's history and clinical examination, the tentative diagnosis of buccal ulceration was made as follows: 1) traumatic lesion associated with mucosal dryness; 2) erosive type of oral lichen planus. With the application of further diagnostic tests, initial treatment for the mucosal lesion was performed to alleviate the patient's chief complaint. The trial therapy included application of prednisolone and amoxicillin rinse and 0.05\% fluocinonide ointment. In order to moisturize the mouth, an oral moisturizing gel with carboxymethylcellulose was also added. At the 1-week follow-up visit, most of the lesions in the buccal mucosa were observed to have subsided. Additional laboratory tests were performed to determine the cause of xerostomia. Serologic studies revealed elevated levels of erythrocyte sedimentation rate, positive rheumatoid factor (RF), antinuclear antibody (ANA), and anti-SSA (Ro) in serum. A mixed pattern of ANA, nuclear speckled, and nucleolar, was observed. The overlapping related disease of both pattern was SS. The tests confirmed that human immunodeficiency virus and hepatitis $C$ were negative.

Further, LSGB was performed on the right lower lip for a definitive diagnosis. Five minor salivary glands were collected and three of them were sampled. On the slides, glandular surface area was measured to be $5.6 \mathrm{~mm}^{2}$. Immunohistochemistry was performed to localize foci and a total of 8 foci were found (Fig. 2, 3). Focus score was calculated as the number of foci per $4 \mathrm{~mm}^{2}$ area, and determined

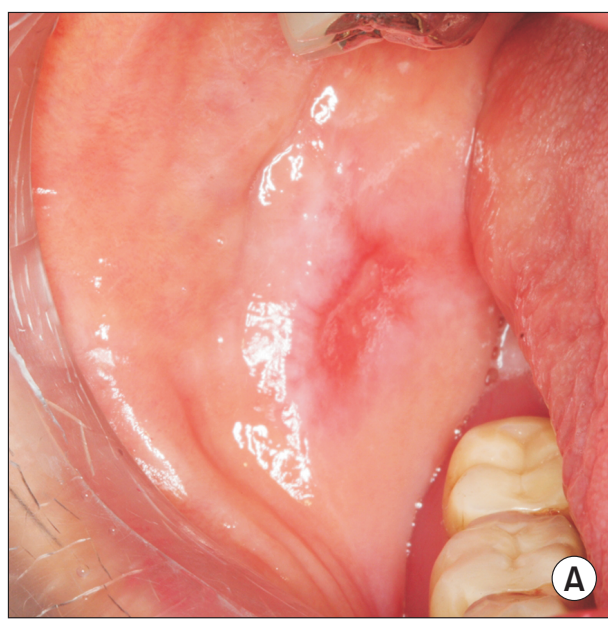

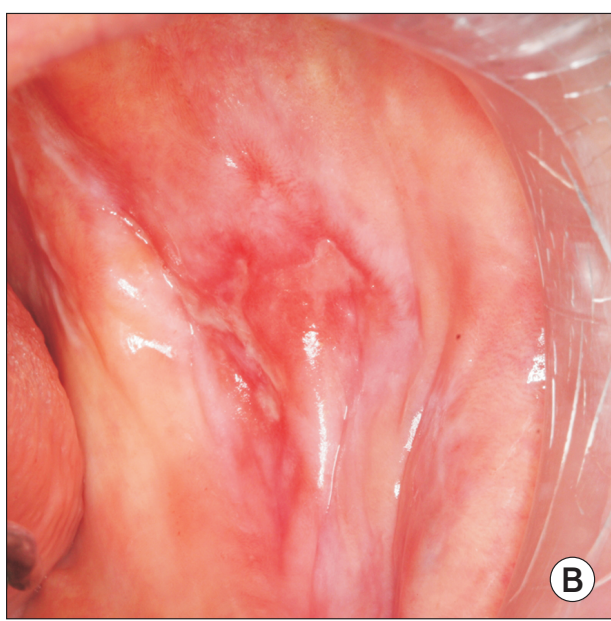

Fig. 1. Intraoral photograph of buccal mucosa showing bilateral ulcerated lesion surrounded by an erythematous border. (A) Right, (B) left. 


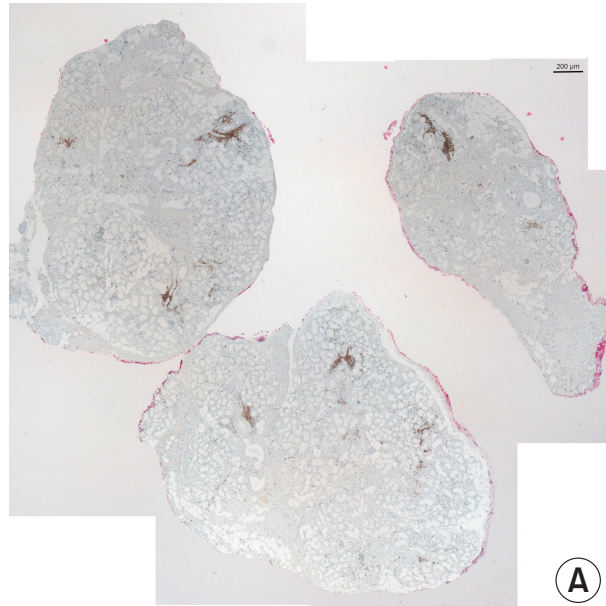

(A)

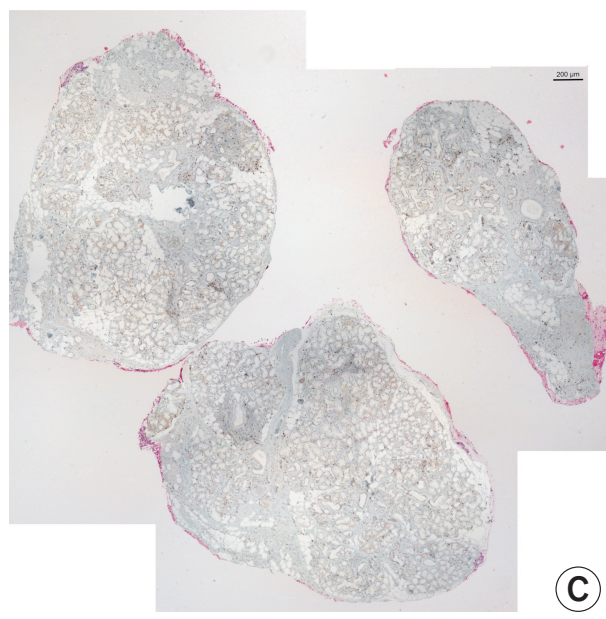

(C)

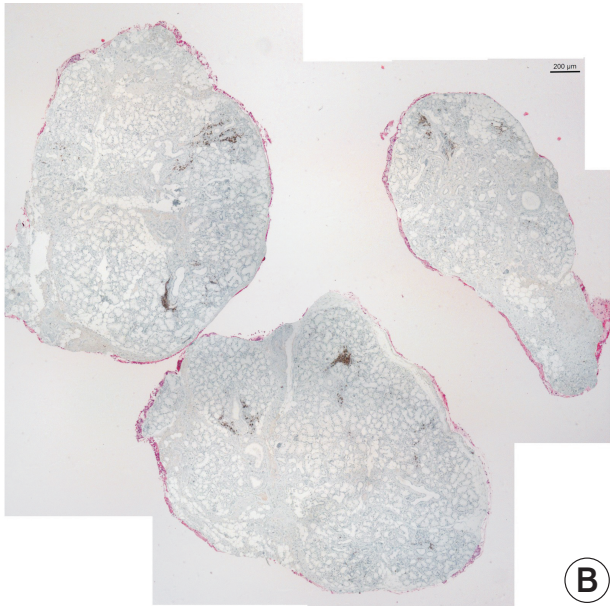

(B)

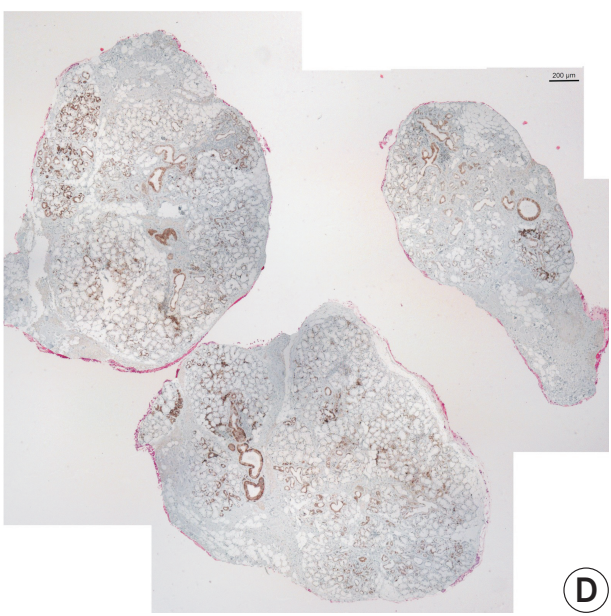

Fig. 2. Immunolocalization of $\mathrm{CD} 3(\mathrm{~A})$, CD20 (B), CD68 (C) and CD138 (D). Stained with immunohistochemistry, original magnification $\times 40$. to be 5.71. Analysis of immunohistochemistry was confirmed by an oral pathologist.

The diagnostic criteria presented by ACR/EULAR in 2016 was met as follows: focal score of 5.71, positive anti-SSA (Ro), 3 points each, and $0 \mathrm{~mL} / 10$ min of unstimulated total salivation 1 point. Total score was calculated to be 7 . Since the total score was more than 4 , the patient was diagnosed with primary SS.

The patient was referred to the department of rheumatology to examine involvement of other organs. In the Department of Oral Medicine, the administration of pilocarpine (Salagen tablet; MGI PHARMA INC., Bloomington, MN, USA) was tried for about 6 months. Salagen treatment was started at $10 \mathrm{mg} /$ day and gradually increased to 20 $\mathrm{mg} /$ day but her salivation did not increase. Salivary scintigraphy was performed to evaluate the function of major salivary glands. The medication was discontinued with her consent since the result revealed the salivary glands had been destroyed too much to respond to Salagen. Regardless of Salagen administration, artificial saliva was constantly provided as palliative therapy. During the course of the follow-up of about 1.5 years to treat dry mouth, the patient did not complain about recurrence of any oral mucosal pain. The patient is still being regularly monitored for oral management of xerostomia at the Kyungpook National University Dental Hospital.

\section{DISCUSSION}

SS is considered a systemic autoimmune disease caused by dysregulation of T and B lymphocytes [5,6]. Patients may have a variety of clinical symptoms depending on the site of lymphocytic invasion [7]. CD4+ T lymphocytes infiltrate and cause damage to fluid-secreting glands, such as salivary glands and lacrimal glands, gradually reducing the volume, and quality of saliva and tears, which are 


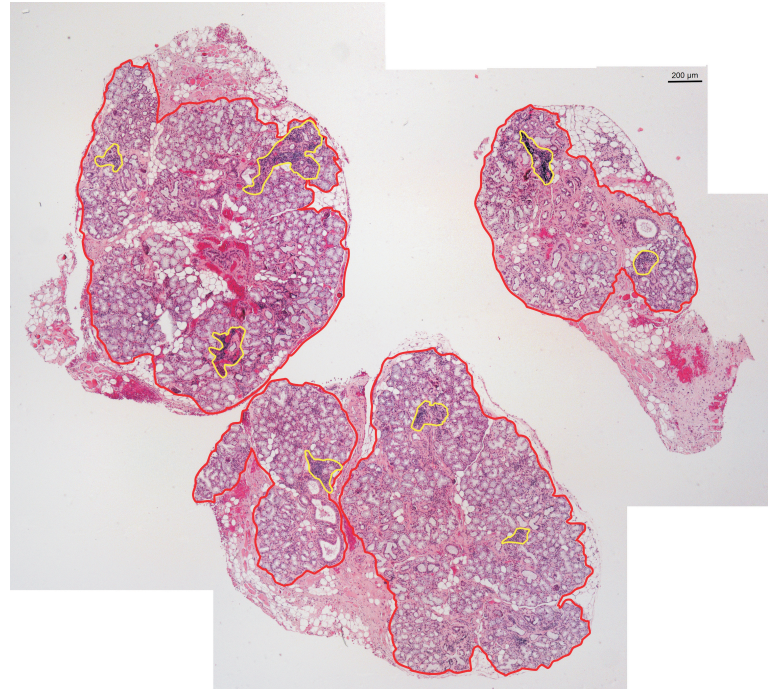

Fig. 3. Histological findings. Glandular surface area in red lines, foci in yellow lines. In this slide, focus score was 5.71. Stained with H\&E, original magnification $\times 40$.

considered the most common symptoms. The disease can affect the nervous system, gastrointestinal system, lungs, genital organ, blood vessels, and kidney in addition to exocrine glands, resulting in chronic fatigue, joint pain, and neuropathic pain [8]. Without proper diagnosis and treatment, patients may have serious complications such as scarred cornea, tooth loss, internal organ involvement, and non-Hodgkin's lymphoma (NHL) [9]. All these symptoms can affect the patient's mental health and lower his/her quality of life.

It can be difficult for clinicians to determine the exact cause of dry mouth. Dry mouth can result from a variety of causes including medication [10], head and neck radiation therapy [11], chemotherapy [12], uncontrolled diabetes [13], as well as SS. Furthermore, systemic manifestation of SS may often be mistaken for menopause, lupus, rheumatoid arthritis, fibromyalgia, multiple sclerosis or as a side effect of medication [1]. Because not all symptoms appear simultaneously, clinicians only focus on individual symptoms and may not be aware of the presence of the underlying systemic diseases. SS may be suspected when dry mouth and the related symptoms are accompanied by systemic symptoms such as dry eyes and joint pain. In this case, the patient's medical history revealed that she was on medication for chronic arthritis in her hands and knees for 20 years. This information gave us a clue about SS.
Dry mouth due to SS can range from mild oral discomfort to severe conditions that can damage the patient's oral health, diet, and quality of life. Primary symptoms of dry mouth include difficulty in tasting, chewing, swallowing, and speaking. Dry and cracked tongue and dry and brittle oral mucosa are also possible symptoms [1,3]. Oral complications include tooth decay, periodontitis, tooth sensitivity, mucositis, traumatic oral lesions, and oral infections such as candidiasis $[1,3]$. Dentists are likely to be the first clinicians to detect dry mouth and the related symptoms caused by dry mucous membranes. Clinicians should be aware that dryness will reduce the lubricity of the mucous membrane, possibly leading to frictional irritation, which can manifest as forms of painful inflammation of the mucous membrane. In this case, the chief complaint of oral mucosal pain, caused by dryness could have been confused with soft tissue disorders such as oral lichen planus. However, oral mucosal pain was resolved with treatment of dry mouth.

Like most other autoimmune diseases, SS is difficult to treat or limit progression with a single medication. Since the disease affects each patient differently, treatment plans must be optimized. Proper oral management of SS must focus on relieving symptoms and preventing the occurrence of complications caused by inadequate saliva production. These managing treatments do not seem to change the progression of the disease.

Clinical trials for SS provide patients with the opportunity to potentially benefit from lifestyle changes such as drugs, medical devices or procedures, and even diet or exercise. It is important to classify patients with standardized diagnostic criteria. The diagnostic criteria used in trial protocol may differ from the one used in clinical environment in certain cases.

According to Shiboski et al. [14], 11 sets of diagnostic criteria for diagnosing SS were published during the period from 1965 to 2002, but none of them were approved by ACR or EULAR. One of the most widely accepted diagnostic criteria to date was published by the AmericanEuropean Consensus Group (AECG) in 2002 (Table 1) [15]. However, it has been criticized for its strong dependency on the patient's subjective symptoms, and lack of diagnostic relevance of Schirmer's test and rose bengal score [16]. Moreover, unstimulated total saliva flow, un-anesthetized 
Table 1. Revised international classification criteria for Sjögren's syndrome, proposed by American - European Consensus Group in 2002

I. Ocular symptoms: a positive response to at least one of the following questions

1. Have you had daily, persistent, troublesome dry eyes for more than 3 months?

2. Do you have a recurrent sensation of sand or gravel in the eyes?

3. Do you use tear substitutes more than three times a day?

II. Oral symptoms: a positive response to at least one of the following questions

1. Have you had a daily feeling of dry mouth for more than 3 months?

2. Have you had recurrent or persistent swollen salivary glands as an adult?

3. Do you frequently drink liquids to aid in swallowing dry food?

III. Ocular signs: that is, objective evidence of ocular involvement defined as a positive result for at least one of the following two tests

1. Schirmer's test, performed without anesthesia ( $<5 \mathrm{~mm}$ in 5 minutes).

2. Rose bengal score or other ocular dye score ( $>4$ according to van Bijsterveld's scoring system).

IV. Histopathology: in minor salivary glands (obtained through normal-appearing mucosa) focal lymphocytic sialoadenitis, evaluated by an expert histopathologist, with a focus score $>1$, defined as a number of lymphocytic foci (which are adjacent to normal-appearing mucous acini and contain more than 50 lymphocytes) per $4 \mathrm{~mm}^{2}$ of glandular tissue.

V. Salivary gland involvement: objective evidence of salivary gland involvement defined by a positive result for at least one of the following diagnostic tests

1. Unstimulated whole salivary flow ( $<1.5 \mathrm{~mL}$ in 15 minutes).

2. Parotid sialography showing the presence of diffuse sialectasias (punctate, cavitary, or destructive pattern), without evidence of obstruction in the major ducts.

3. Salivary scintigraphy showing delayed uptake, reduced concentration, and/or delayed excretion of tracer.

VI. Autoantibodies: presence in the serum of the following autoantibodies

1. Antibodies to Ro (SSA) or La (SSB) antigens, or both

For primary Sjögren's syndrome (SS): in patients without any potentially associated disease, primary SS may be defined as follows

a. The presence of any 4 of these 6 criteria is indicative of primary SS, as long as either criterion IV (histopathology) or VI (serology) is positive.

b. The presence of any 3 of the 4 objective criteria items (that is, items III, IV, V, VI)

c. The classification tree procedure represents a valid alternative method for classification, although it should be more properly used in clinical-epidemiological survey.

For secondary SS: in patients with a potentially associated disease (for instance, another well-defined connective tissue disease), the presence of item I or item II plus any 2 from among items III, IV, and V may be considered as indicative of secondary SS

Exclusion criteria:

Past head and neck radiation treatment

Hepatitis C infection

Acquired immunodeficiency syndrome (AIDS)

Pre-existing lymphoma

Sarcoidosis

Graft versus host disease

Use of anticholinergic drugs (since a time shorter than 4-fold the half-life of the drug)

SSA, Sjögren's-syndrome-related antigen A; SSB, Sjögren's-syndrome-related antigen B.

Adapted from the article of Vitali et al. (Ann Rheum Dis 2002;61:554-558) [15] with original copyright holder's permission.

Schirmer's test, and salivary scintigraphy were useful for evaluating salivary or tear function, but they showed low diagnostic specificity for SS.

In 2012, ACR proposed a new diagnostic criteria with three objective items of autoantibody, ocular stain score, and LSGB (Table 2) [16]. This ACR criteria differ from the AECG criteria in several aspects; (1) It excludes patient's subjective ocular and oral dryness. There are no functional or morphological tests of the salivary glands. (2) It uses the new ocular stain score as the sole criteria for eye involvement. (3) Positive ANA and RF can be an alternative to anti-SSA (Ro) or -SSB (La) antibody positivity as evaluation of systemic autoimmunity [17]. Since the ACR criteria was established to classify patients for clinical trial, it is more stringent for definitive diagnosis of SS than the AECG criteria [16].

In the following studies, ACR criteria turned out to show more false-negatives than the AECG criteria [17,18]. Low detection rate of diagnosis could be more influential to patients than false-positives. In addition, despite the low diagnostic relevance of ocular staining score, it has similar diagnostic importance as serology and focus scores [16]. There is no credible evidence that the ACR criteria is more valuable than the AECG criteria in clinical or biological aspect 
Table 2. American College of Rheumatology's classification criteria in 2012

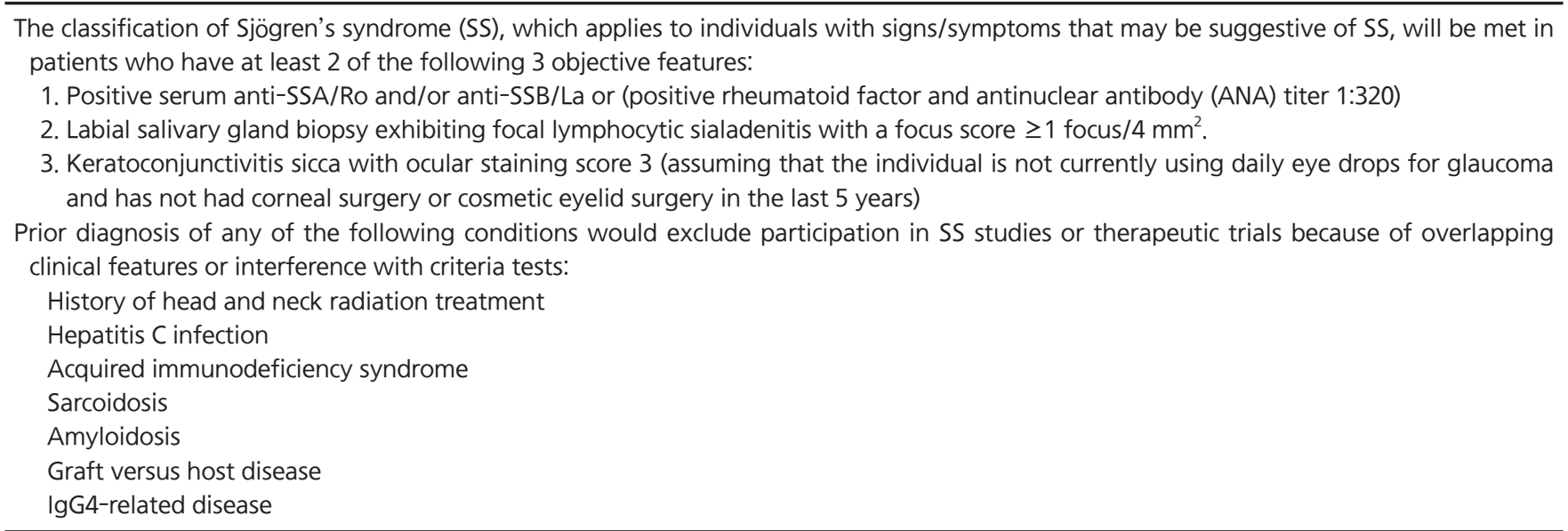

SSA, Sjögren's-syndrome-related antigen A; SSB, Sjögren's-syndrome-related antigen B; IgG4, immunoglobulin G4.

Adapted from the article of Shiboski et al. (Arthritis Care Res [Hoboken] 2012;64:475-487) [16] with original copyright holder's permission.

Table 3. American College of Rheumatology/European League against Rheumatism classification criteria for primary Sjögren's syndrome in 2016

\begin{tabular}{lc}
\hline \multicolumn{1}{c}{ Item } & Weight/score \\
\hline Labial salivary gland with focal lymphocytic sialadenitis and focus score of $\geq 1$ & 3 \\
Anti-SSA/Ro positive & 3 \\
Ocular staining score $\geq 5$ (or van Bijsterfeld score $\geq 4$ ) on at least one eye & 1 \\
Schirmer $\leq 5 \mathrm{~mm} / 5$ min on at least one eye & 1 \\
Unstimulated whole saliva flow rate $\leq 0.1 \mathrm{~mL} / \mathrm{min}$ & 1 \\
Exclusion criteria include prior diagnosis of any of the following conditions, which would exclude diagnosis of SS and participation in SS \\
studies or therapeutic trials because of overlapping clinical features or interference with criteria tests: \\
1) History of head and neck radiation treatment \\
2) Active hepatitis C infection (with confirmation by polymerase chain reaction \\
3) Acquired immunodeficiency syndrome (AIDS) \\
4) Sarcoidosis \\
5) Amyloidosis \\
6) Graft versus host disease \\
7) IgG4-related disease
\end{tabular}

SSA, Sjögren's-syndrome-related antigen A; IgG4, immunoglobulin G4.

The classification of primary Sjögren's syndrome (pSS) applies to any individual who meets the inclusion criteria, does not have any condition listed as exclusion criteria, and who has a score $\geq 4$ when summing the weights from the above items.

Adapted from the article of Shiboski et al. (Ann Rheum Dis 2017;76: 9-16) [14] with original copyright holder's permission.

[18]. It is necessary to develop a new, more precise diagnostic criteria to overcome limitations of the previous ones.

The latest diagnostic criteria were approved by ACR/ EULAR in 2016 (Table 3) [14]. It includes only items that are objective as well as easy to perform in clinical settings. The new diagnostic criteria ranks the existing test items by cohort verification, and the weight of each item is allocated accordingly. Focus score of LSGB and positivity of anti-SSA (Ro) are assigned 3 points, respectively, indicating greater significance. Ocular staining score, Schirmer's test, and saliva flow test are assigned 1 point each. This criterion is satisfied when the sum of the score is 4 or more. Subjective oral and ocular symptoms are not given points, but they are still considered important in raising clinical suspicion and providing suitable guidance for diagnostic tests required for SS. As sialography and salivary scintigraphy in the AECG criteria were excluded, oral examination is now assessed by LSGB and unstimulated salivary flow rate. The sensitivity and specificity of the new criteria are 96\% (95\% confidence interval [CI] 92\%-98\%) and 95\% (95\% CI 92\%-97\%), respectively, which indicates high diagnostic accuracy [14].

The greatest strength of the ACR/EULAR criteria is the 
recognition of the systemic characteristics of SS. It can classify the patients with extra-glandular symptoms without oral or ocular dryness, and with B cell activation markers [4]. The use of this criteria will likely help diagnose the disease in younger patients before they develop glandular symptoms, or in nordic patients who have been found to experience more than two times less dry eye than other SS patients [19]. This classification criteria can potentially lead to earlier recognition of patients with SS. Given the purpose of the 2016 diagnostic criteria to identify patients for clinical trials, young SS patients will particularly benefit from new treatment options. Furthermore, use of the weighted scoring system will possibly minimize the intrinsic differences between test items, thus reducing the inconsistencies caused by use of the current criteria. Finally, the new score summing system can be adapted to conform to the variability associated with the use of new diagnostic methods such as ultrasound imaging or improved serological analysis by adding new items or modifying the weighted scores [4].

Excessive autoantibodies are found in the blood of patients with autoimmune diseases such as SS. In SS, in particular, anti-SSA (Ro) is included in the ACR/EULAR diagnostic criteria and assigned 3 points, the highest weight. RF, and anti-SSB (La) are excluded due to the lack of specificity. Because of the simplicity of blood tests, it can be useful in diagnosing SS and other autoimmune disorders. However, blood tests should not be used as a screening test to diagnose SS. It has been reported that up to 50\% of SS patients are sero-negative [20-22]. Also, these antibodies are nonspecific to SS patients, and can be found in patients with other rheumatic diseases or even in healthy individuals. So, in the case of SS depending only on serologic tests for diagnosis may cause a delay in final diagnosis [20].

Like serology, the LSGB is given the maximum weight of 3 points. Positive LSGB can contribute to final diagnosis of SS only when the patient is positive for one of the clinical symptoms. Rheumatologists are primarily responsible for diagnosing and managing SS, but dentists should also be able to perform diagnostic tests. Since dentists are familiar with surgical procedures and capable of managing LSGB, it can be easily performed. LSGB is a minimally invasive procedure unlike tooth implantation or extraction. Most of its complications such as bleeding, pain, and inflammation are transient and easily manageable [23].

LSGB is not only a diagnostic process, but also can be used as a tool to predict the prognosis of a disease. The most serious complication associated with primary SS is development of lymphoproliferative disorders, mainly NHL [24]. The risk of NHL in primary SS has been observed to be 6 to20 times higher than the healthy controls $[8,25]$. This fatal complication is predicted to occur in 5\% to $10 \%$ of all primary SS patients [26]. The germinal center-like structures, which is assumed to be a biomarker for predicting NHL can be found in LSGB [26]. SS patients with higher levels of lymphocytic infiltration (e.g., a higher focus score) have been shown to have increased risk of lymphoma [27]. In addition, it is rare but SS-related complications such as myocarditis and pulmonary fibrosis are highly associated with a 2- to 4-fold higher mortality rate [28]. Detecting conditions with a high mortality rate during diagnosis will be effective for closer follow-up and more sophisticated care.

In this case, the patient underwent a blood test, LSGB, salivary flow test, and salivary scintigraphy in the Department of Oral Medicine at Kyungpook National University Dental Hospital, and was diagnosed as primary SS using any of the three diagnostic criteria discussed above. However, depending on the symptoms observed the available diagnostic methods in clinical environment can vary, and diagnosis can differ based on the diagnostic criteria followed. Using the most up-to-date diagnostic criteria may be clinically more effective within a limited resource in some patients.

According to the results of a survey conducted by the SS Foundation, the average diagnosis time of primary SS was more than 6 years in 2012 [29]. However, it was shortened to 2.8 years in 2018. This reduction of diagnosis time could be explained in terms of following 2 aspects: first, use of the new criteria has enhanced the accuracy of disease identification. Second, many clinicians especially those in rheumatology, ophthalmology, and dentistry have become more familiar with SS. Dentists may contribute to improving quality of life of SS patients with the related oral symptoms through careful diagnosis using the latest diagnostic criteria.

Dentists are likely to encounter patients with dry mouth, a major clinical symptom of SS. Even if the patient's main 
complaint is not dry mouth, clinical symptoms and signs that suggest SS, such as dry mucosal surfaces, soft tissue pain, and multiple dental caries may be observed. Standardized diagnostic criteria enable early diagnosis and appropriate management that may keep the patients from serious complications. The most up-to-date 2016 ACR/ EULAR diagnostic criteria require a LSGB showing the characteristic pattern of inflammation or presence of anti-SSA (Ro) antibodies. The advantage of using the latest criteria is that it can help with early identification of patients who have not yet developed dry symptoms. Dentists, particularly oral medicine specialists, should be aware of oral complications of xerostomia caused by SS and not hesitate to apply the latest diagnostic criteria to patients with suspected symptoms.

\section{CONFLICT OF INTEREST}

No potential conflict of interest relevant to this article was reported.

\section{ORCID}

\author{
Ji-Young Lee \\ https://orcid.org/0000-0003-4116-1188 \\ Jin-Seok Byun \\ https://orcid.org/0000-0002-6182-1238 \\ Jae-Kwang Jung \\ https://orcid.org/0000-0003-3099-8097 \\ Jae-Kap Choi \\ https://orcid.org/0000-0001-6773-7507
}

\section{REFERENCES}

1. Kassan SS, Moutsopoulos HM. Clinical manifestations and early diagnosis of Sjögren syndrome. Arch Intern Med 2004;164:12751284.

2. Brandt JE, Priori R, Valesini G, Fairweather D. Sex differences in Sjögren's syndrome: a comprehensive review of immune mechanisms. Biol Sex Differ 2015;6:19.

3. Cartee DL, Maker S, Dalonges D, Manski MC. Sjögren's syndrome: oral manifestations and treatment, a dental perspective. J Dent Hyg 2015;89:365-371.

4. Franceschini F, Cavazzana I, Andreoli L, Tincani A. The 2016 classification criteria for primary Sjogren's syndrome: what's new? BMC Med 2017;15:69.

5. Ramos-Casals M, Tzioufas AG, Font J. Primary Sjögren's syndrome: new clinical and therapeutic concepts. Ann Rheum Dis 2005;64:347-354.

6. Pertovaara M, Korpela M, Uusitalo H, et al. Clinical follow up study of 87 patients with sicca symptoms (dryness of eyes or mouth, or both). Ann Rheum Dis 1999;58:423-427.

7. Baer AN, Walitt B. Sjögren syndrome and other causes of sicca in older adults. Clin Geriatr Med 2017;33:87-103.

8. Zintzaras E, Voulgarelis M, Moutsopoulos HM. The risk of lymphoma development in autoimmune diseases: a meta-analysis. Arch Intern Med 2005;165:2337-2344.

9. Mariette X, Criswell LA. Primary Sjögren's syndrome. N Engl J Med 2018;378:931-939.

10. Villa A, Connell CL, Abati S. Diagnosis and management of xerostomia and hyposalivation. Ther Clin Risk Manag 2014;11:45-51.

11. Ship JA. Diagnosing, managing, and preventing salivary gland disorders. Oral Dis 2002;8:77-89.

12. Cohen-Brown G, Ship JA. Diagnosis and treatment of salivary gland disorders. Quintessence Int 2004;35:108-123.

13. López-Pintor RM, Casañas E, González-Serrano J, et al. Xerostomia, hyposalivation, and salivary flow in diabetes patients. J Diabetes Res 2016;2016:4372852.

14. Shiboski CH, Shiboski SC, Seror R, et al. 2016 American College of Rheumatology/European League Against Rheumatism classification criteria for primary Sjögren's syndrome: a consensus and data-driven methodology involving three international patient cohorts. Ann Rheum Dis 2017;76:9-16.

15. Vitali C, Bombardieri S, Jonsson R, et al. Classification criteria for Sjögren's syndrome: a revised version of the European criteria proposed by the American-European Consensus Group. Ann Rheum Dis 2002;61:554-558.

16. Shiboski SC, Shiboski CH, Criswell L, et al. American College of Rheumatology classification criteria for Sjögren's syndrome: a data-driven, expert consensus approach in the Sjögren's International Collaborative Clinical Alliance cohort. Arthritis Care Res (Hoboken) 2012;64:475-487.

17. Cornec D, Saraux A, Cochener B, et al. Level of agreement between 2002 American-European Consensus Group and 2012 American College of Rheumatology classification criteria for Sjögren's syndrome and reasons for discrepancies. Arthritis Res Ther 2014;16:R74.

18. Rasmussen A, Ice JA, Li H, et al. Comparison of the AmericanEuropean Consensus Group Sjogren's syndrome classification criteria to newly proposed American College of Rheumatology criteria in a large, carefully characterised sicca cohort. Ann Rheum Dis 2014;73:31-38

19. Kuryata O, Lysunets T, Karavanska I, Semenov V. Duration till diagnosis and clinical profile of Sjögren's syndrome: data from real clinical practice in a single-center cohort. Egypt Rheumatol 2020;42:41-46.

20. Mathews PM, Hahn S, Hessen M, et al. Ocular complications of primary Sjögren syndrome in men. Am J Ophthalmol 2015;160:447-452.e1.

21. Fayyaz A, Kurien BT, Scofield RH. Autoantibodies in Sjögren's 
syndrome. Rheum Dis Clin North Am 2016;42:419-434.

22. Baer AN, Gutierrez L, DeMarco MM, et al. Prevalence of seronegative Sjogren's syndrome: a comparative study from the Sjogren's International Collaborative Clinical Alliance (SICCA) cohort. Paper presented at: 2013 ACR/ARHP Annual Meeting; 2013 Oct 2530; San Diego, USA. pp. S226.

23. Varela-Centelles P, Seoane-Romero JM, Sánchez-Sánchez M, González-Mosquera A, Diz-Dios P, Seoane J. Minor salivary gland biopsy in Sjögren's syndrome: a review and introduction of a new tool to ease the procedure. Med Oral Patol Oral Cir Bucal 2014;19:e20-e23.

24. Kruszka P, O’Brian RJ. Diagnosis and management of Sjögren syndrome. Am Fam Physician 2009;79:465-470.

25. Smedby KE, Hjalgrim H, Askling J, et al. Autoimmune and chronic inflammatory disorders and risk of non-Hodgkin lymphoma by subtype. J Natl Cancer Inst 2006;98:51-60.
26. Theander E, Vasaitis L, Baecklund E, et al. Lymphoid organisation in labial salivary gland biopsies is a possible predictor for the development of malignant lymphoma in primary Sjögren's syndrome. Ann Rheum Dis 2011;70:1363-1368.

27. Risselada AP, Kruize AA, Goldschmeding R, Lafeber FP, Bijlsma JW, van Roon JA. The prognostic value of routinely performed minor salivary gland assessments in primary Sjögren's syndrome. Ann Rheum Dis 2014;73:1537-1540.

28. Palm 0, Garen T, Berge Enger T, et al. Clinical pulmonary involvement in primary Sjogren's syndrome: prevalence, quality of life and mortality--a retrospective study based on registry data. Rheumatology (Oxford) 2013;52:173-179.

29. Sjögren's Foundation. Breakthrough goal. Sjögren's Foundation accomplishes 5-year breakthrough goal [Internet]. Reston: Sjögren's Foundation; c2018 [cited 2021 May 4]. Available from: https://www.sjogrens.org/about-us/history/breakthrough-goal. 